\section{(2) OPEN ACCESS}

\title{
Structural weakening of the colonic mucus barrier is an early event in ulcerative colitis pathogenesis
}

\author{
Sjoerd van der Post, ${ }^{1}$ Karolina S Jabbar, ${ }^{1}{ }^{1} 2$ George Birchenough, ${ }^{1}$ Liisa Arike, ${ }^{1}$ \\ Noreen Akhtar, ${ }^{1}$ Henrik Sjovall, ${ }^{2}$ Malin E V Johansson, ${ }^{1}$ Gunnar C Hansson (i) ${ }^{1}$
}

\begin{abstract}
- Additional material is published online only. To view please visit the journal online (http://dx.doi.org/10.1136/ gutjnl-2018-317571).

${ }^{1}$ Department of Medical Biochemistry, University of Gothenburg, Gothenburg, Sweden

${ }^{2}$ Department of

Gastroenterology, Sahlgrenska University Hospital, Gothenburg, Sweden
\end{abstract}

Correspondence to gunnar.hansson@medkem.gu.se

SvdP and KSJ contributed equally.

SvdP and KSJ shared first author.

Received 11 September 2018 Revised 8 February 2019

Accepted 5 March 2019 Published Online First 26 March 2019
Dr Gunnar C Hansson;

\begin{abstract}
Objective The colonic inner mucus layer protects us from pathogens and commensal-induced inflammation, and has been shown to be defective in active UC. The aim of this study was to determine the underlying compositional alterations, their molecular background and potential contribution to UC pathogenesis. Design In this single-centre case-control study, sigmoid colon biopsies were obtained from patients with UC with ongoing inflammation $(n=36)$ or in remission $(n=28)$, and from 47 patients without colonic disease. Mucus samples were collected from biopsies ex vivo, and their protein composition analysed by nanoliquid chromatography-tandem mass spectrometry. Mucus penetrability and goblet cell responses to microbial stimulus were assessed in a subset of patients.
\end{abstract}

Results The core mucus proteome was found to consist of a small set of 29 secreted/transmembrane proteins. In active UC, major structural mucus components including the mucin MUC2 $(p<0.0001)$ were reduced, also in non-inflamed segments. Active UC was associated with decreased numbers of sentinel goblet cells and attenuation of the goblet cell secretory response to microbial challenge. Abnormal penetrability of the inner mucus layer was observed in a subset of patients with UC (12/40; 30\%). Proteomic alterations in penetrable mucus samples included a reduction of the SLC26A3 apical membrane anion exchanger, which supplies bicarbonate required for colonic mucin barrier formation.

Conclusion Core mucus structural components were reduced in active UC. These alterations were associated with attenuation of the goblet cell secretory response to microbial challenge, but occurred independent of local inflammation. Thus, mucus abnormalities are likely to contribute to UC pathogenesis.

\section{INTRODUCTION}

Check for updates

(C) Author(s) (or their employer(s)) 2019. Re-use permitted under CC BY-NC. No commercial re-use. See rights and permissions. Published by BMJ.

To cite: van der Post $S$ Jabbar KS, Birchenough G, et al. Gut

2019:68:2142-2151.
$\mathrm{UC}$ is the most common IBD, and its incidence is increasing. ${ }^{1}$ Although the pathogenesis is incompletely understood, there is consensus that inappropriate activation of the immune system by commensal bacteria underlies the disease. ${ }^{1}$ Normally, a secreted, attached and stratified mucus barrier allows us to reap the benefits of abundant and diverse luminal microbiota, while shielding the epithelium and underlying immune system from bacterial contact and erroneous activation. $^{2}$ The importance of mucus protection is

\section{Significance of this study}

What is already known on this subject?

- Abnormalities of the mucus system, including emptying of upper crypt goblet cells, visible mucus in stool and bacterial penetration of the inner mucus layer, have previously been described in active UC.

What are the new findings?

- Structural weakening of the mucus barrier, with a reduction of core components MUC2 and FCGBP, occurs prior to the onset of inflammation in UC.

- Mucus barrier weakening and failure is associated with exhaustion of the sentinel goblet cell response to microbes.

How might it impact on clinical practice in the foreseeable future?

- Our observations indicate that weakening of the mucus barrier is common in active UC, occurs independent of local inflammation and likely plays a role in UC pathogenesis. Thus, targeting of the underlying mechanisms identified in this study may prevent relapses and prolong remission periods in patients with UC.

evident from studies of mice lacking the main colonic mucin, Muc2, and thus a functional mucus layer. These mice develop severe colitis, and subsequently colon cancer, likely due to increased epithelial exposure to bacteria. ${ }^{2-4}$ The role of the microbiota is supported by various mouse models that ordinarily develop spontaneous colitis, but are protected in germ-free conditions or during antibiotic treatment. ${ }^{56}$

Despite the crucial function of the intestinal mucus at the host-microbial interface, its composition has remained largely unknown. Presently, through the combination of an experimental set-up where mucus could be secreted, visualised and collected from ex vivo endoscopic biopsies, and modern mass spectrometry (MS) methods allowing for the analysis of minimal sample quantities, we were able to determine the protein constituents of the human colonic mucus barrier in a study of over 100 patients with UC and individuals without colonic disease. 
While abnormalities of mucus secretion and barrier function are common in UC, the underlying mechanisms and their temporal and causal relationship with the inflammation are poorly understood. ${ }^{16}$ Herein, we report on alterations of the colon mucus barrier composition in UC, and show that structural weakening occurs independent of local inflammation. Furthermore, using fluorescent beads as surrogate markers for bacteria, we determined the compositional characteristics of penetrable mucus. ${ }^{6}$ Finally, evidence is presented that suggests a possible causative role of primary mucus barrier failure for a subgroup of patients with UC.

\section{METHODS}

\section{Recruitment of patients and sample collection}

Patients with UC and individuals without suspected IBD $\geq 18$ years, who were referred to Sahlgrenska University Hospital (Gothenburg, Sweden) for colonoscopy, were eligible for inclusion; subject to the provision of written informed consent. Patients with macroscopic/microscopic evidence of colon pathology other than UC were excluded. Clinically active UC was defined as two, or more, of the following: Mayo score $\geq 3$, endoscopic Mayo score $\geq 1$, or Sandborn histological score $\geq 2 .{ }^{78}$ Eight biopsies were obtained from the sigmoid colon, two of which were used for mucus collection. In six additional patients without IBD, two biopsies were taken from macroscopically normal ileum.

Our predefined target sample size was 100 individuals, with a relatively equal distribution of controls, UC in remission and patients with active UC. Individuals with mild, rather than severe, inflammation were preferentially selected.

\section{Mucus collection}

Biopsies were transported in oxygenated, ice-cold Krebs buffer and mounted in our in-house developed ex vivo perfusion chambers. ${ }^{9}$ Immediately after mounting, a thin mucus layer can be visualised by sprinkling charcoal onto the apical surface. Biopsies were kept for 1 hour in the chamber with continuous apical and basolateral supply of nutrients and oxygen; during this period the mucus typically grew to at least double thickness. Subsequently, mucus was collected by gentle scraping and stored at $-80^{\circ} \mathrm{C}$.

\section{Sample preparation, MS and data processing}

Preparation of mucus samples and MS analysis were performed as detailed in the online supplementary methods. Nanoliquid chromatography-tandem MS was carried out on an LTQ-Orbitrap XL (Thermo); targeted quantification of MUC2 by parallel reaction monitoring on a Q-Exactive instrument (Thermo). ${ }^{10}$ Proteins were identified through searches against the human Swiss-Prot database (v2014 1), using Andromeda integrated into MaxQuant (V.1.3.0.5). ${ }^{\overline{1}}$ Protein abundance factors were calculated by normalising individual peptide intensities against the total intensity of each patient sample. Absolute quantification of MUC2 was performed with Skyline (V.3.6.0.1). ${ }^{12}$

\section{Stimulation of colon biopsies with Toll-like receptor 2 ligand $\mathrm{P}_{3} \mathrm{CSK}_{4}$ and quantification of sentinel goblet cells}

The goblet cell secretory response to Toll-like receptor (TLR) $1 / 2$ ligand $\mathrm{P}_{3} \mathrm{CSK}_{4}$ was assessed in ex vivo human biopsies, as previously described for mouse tissue. ${ }^{13}$ Sentinel goblet cells were quantified by preparing biopsy whole mounts and identifying endocytotic goblet cells as previously described. ${ }^{13}$
Table 1 Patient demographics and clinical characteristics

\begin{tabular}{|c|c|c|c|}
\hline & Controls & Active UC & $\begin{array}{l}\text { UC in } \\
\text { remission }\end{array}$ \\
\hline Patients & 47 & 36 & 28 \\
\hline Females, n (\%) & $22(47)$ & $15(42)$ & $14(50)$ \\
\hline Age: median; range & $59(23-87)$ & $38(18-70)$ & $54(28-75)$ \\
\hline Active smokers, n (\%) & $13(28)$ & $2(6)$ & $2(7)$ \\
\hline PSC, $n(\%)$ & $1(2)$ & $4(11)$ & $2(7)$ \\
\hline Years since UC diagnosis: median; range & NA & $12(0-34)$ & $21(5-48)$ \\
\hline Mayo endoscopy score* $0, n(\%)$ & NA & $6(17)$ & $27(96)$ \\
\hline Mayo endoscopy score* 1, n (\%) & NA & $22(61)$ & $1(4)$ \\
\hline Mayo endoscopy score* $2, n(\%)$ & NA & $7(19)$ & $0(0)$ \\
\hline Mayo endoscopy score* 3, n (\%) & NA & $1(3)$ & $0(0)$ \\
\hline Sandborn histological scoret $0, n(\%)$ & NA & $9(27)$ & $14(54)$ \\
\hline Sandborn histological scoret $1, n(\%)$ & NA & $9(27)$ & $9(35)$ \\
\hline Sandborn histological score† 2, n (\%) & NA & $12(36)$ & $3(12)$ \\
\hline Sandborn histological scoret 3, n (\%) & NA & $3(9)$ & $0(0)$ \\
\hline Medication; 5-ASA, n (\%) & NA & $31(86)$ & $16(57)$ \\
\hline Medication; AZA, n (\%) & NA & $5(14)$ & $1(4)$ \\
\hline Medication; TNF $\alpha$ inhibitor, n (\%) & NA & $1(3)$ & $1(4)$ \\
\hline
\end{tabular}

* Refers to the sigmoid colon.

†Available for 33/36 active UC and 26/28 UC in remission. Refers to the sigmoid colon.

5-ASA, 5-aminosalicylic acid; AZA, azathioprine; NA, not applicable; PSC, primary sclerosing cholangitis; TNF $\alpha$, tumour necrosis factor-alpha.

\section{Immunohistochemistry}

Paraffin-embedded methanol-Carnoy fixed sections from the sigmoid colon were dewaxed and stained with the primary antibodies listed in the online supplementary table S1.

\section{Analysis of mucus penetrability by confocal microscopy}

Biopsies were mounted in a perfusion chamber, and fluorescent beads $(0.5,1$ and $2 \mu \mathrm{m}$ diameter) deposited on the mucus surface. ${ }^{69}$ After $40 \mathrm{~min}$, confocal z-stack images were acquired. The mucus was considered penetrable if there was an accumulation of beads at the epithelial surface, semipenetrable if there was a scattering of beads throughout the inner mucus layer, and impenetrable in the case of a clear separation between beads and epithelium. The mean distance from the epithelium to the 20 most penetrating beads was also determined. Detailed information is provided in the online supplementary methods.

\section{Statistics}

One-way analysis of variance (with Tukey's post hoc test) or Kruskal-Wallis test was used for comparisons of multiple groups; Mann-Whitney U test with Bonferroni adjustment of the significance threshold $(\mathrm{p}<0.05)$ for two-by-two comparisons. P values are two sided. Data analysis was performed using GraphPad Prism (V.6) and R open source software.

\section{RESULTS}

\section{Study population}

Between September 2010 and June 2012, a total of 36 patients with active UC, 28 patients with UC in remission and 47 controls were included (table 1). Indications for colonoscopy for the control group are provided in the online supplementary table S2. Except for polyps and diverticulosis, all controls had normal findings on colonoscopy, and routine histology (performed in $19 / 47$ controls) was invariably normal. 

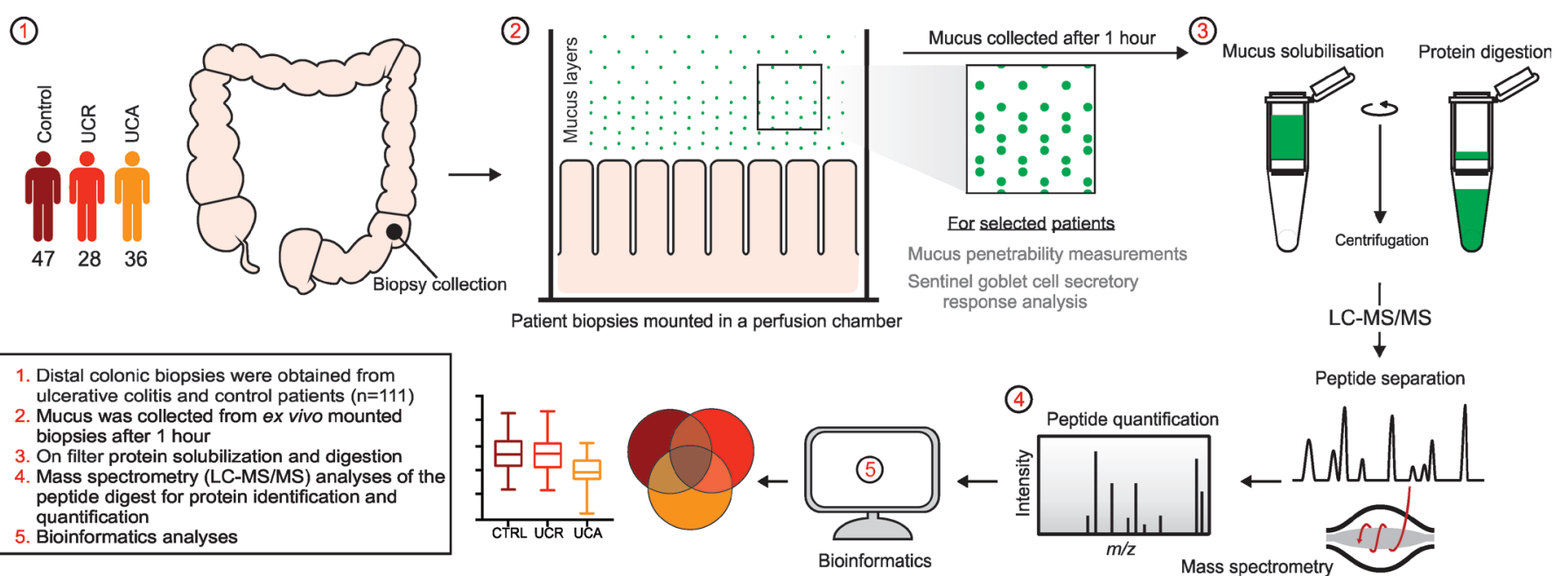

Figure 1 Schematic overview of the study design and workflow of the mass spectrometry analysis of colonic mucus samples. LC-MS/MS, liquid chromatography-tandem mass spectrometry; m/z, mass-to-charge ratio; UC, ulcerative colitis.

Sigmoid biopsies from 10 patients with UC (active: 5; remission: 5) and five controls were used for absolute quantification of MUC2, assessment of sentinel goblet cell numbers and of goblet cell secretory responses to microbial challenge (online supplementary table S3). Additionally, ileal biopsies were obtained from six patients without IBD and with macroscopically normal colon and ileum (online supplementary table S4).

\section{The colon mucus barrier is formed by 29 core proteins}

Mucus samples collected from ex vivo sigmoid colon biopsies were analysed by tandem MS to determine their protein composition, as summarised in figure 1 . The median number of identified proteins was 955 (range 500-1291) for controls and 910 (range 450-1341) for patients with UC. The distribution of protein identifications is illustrated in the online supplementary figure $\mathrm{S} 1$. The core mucus proteome was established from individuals without colon pathology $(n=47)$, based on proteins identified in $>50 \%$ of samples (853 proteins; online supplementary table S5). Epithelial cells are continuously renewed and exfoliated into the mucus, explaining the detection of intracellular proteins (online supplementary figure S2). ${ }^{9}$ However, only proteins carrying a signal peptide for secretion, or annotated to be membrane spanning or lipidated, were considered potential members of the mucus proteome. Next, proteins with motifs that prevent secretion from the endoplasmic reticulum (except AGR2) were excluded, and known contaminants from blood/extracellular fluid removed (online supplementary figure S2). ${ }^{14}{ }^{15}$ This stringent filtering resulted in a shortlist of 71 proteins (online supplementary table S5). Most of these could be grouped into four functional categories: defence response, mucus components, extracellular matrix organisation and glycoprotein biosynthesis (online supplementary figure S3).

Hierarchical clustering of the correlation coefficients for all mucus proteins in all samples is shown in figure 2A. Studies of the correlation between the MUC2 mucin, the main constituent of the intestinal mucus, and other components could reveal additional proteins of particular importance for barrier structure and function. ${ }^{216}$ MUC2 levels correlated most closely with those of the protein FCGBP (Pearson's $r=0.77, p<0.0001$, figure 2B), corroborating previous evidence that these proteins are covalently linked. ${ }^{16}$ Furthermore, there was a strong correlation $(r=0.66, p<0.0001)$ between FCGBP and the protein CLCA1, suggesting that CLCA1 is an important component of the mucus layer in humans, as previously shown for mice (figure 2C). ${ }^{16}$

Finally, manual curation of the shortlist identified several proteins whose known function and cellular origin appeared incompatible with a role in the mucus barrier, for example, collagens and integrins. Their removal resulted in a list of 29 core mucus proteins (table 2).

Certain previously identified or predicted colon mucus components were not detected, including trefoil factors and classical antimicrobial proteins. ${ }^{17-19}$ Trefoil factors are small proteins that may not generate tryptic peptides identifiable by MS. To exclude that the lack of classical antimicrobial proteins was due to technical limitations, we analysed ileal mucus samples from six patients without intestinal pathology (online supplementary tables S4 and S6). Comparing the colonic and ileal mucus proteomes, a number of proteins were found to be unique to the latter. These included Paneth cell-derived antimicrobial proteins, some of which are highly abundant (online supplementary figure S4). ${ }^{18} 19$ Consequently, although these proteins probably pass through the colonic lumen, they are unlikely to form part of its inner mucus layer.

\section{Mucus core structural components are reduced in active UC}

Having defined the healthy colon mucus proteome, we looked for alterations in UC. Eosinophil products were more commonly detected in UC (including remission), and neutrophil proteins during active inflammation. Otherwise, there were no major differences in protein identifications between patient groups (online supplementary figure S5 and table S7). Therefore, we instead compared the relative abundances of the core mucus proteins. Nine out of 29 proteins (31\%) were significantly altered in active UC, seven of which were reduced (figure 3, table 2). These included core structural components MUC2 and FCGBP ( $p<0.0001$ for both) as well as other goblet cell products, such as CLCA1 $(p=0.01)$ and ZG16 $(p=0.001) .^{162021}$ The two proteins that were more abundant in active UC (RNASE3/ ECP and EPX) are both eosinophil derived. RNASE3 was also increased in remission (figure 3).

Mucus thickness is generally reduced in active UC. ${ }^{6}$ However, by normalising the individual protein intensity values to the total intensity of the sample, any effects of sample volume on the results could be neutralised. Increased cell turnover and apoptosis in colitis might suppress the detection of the true 

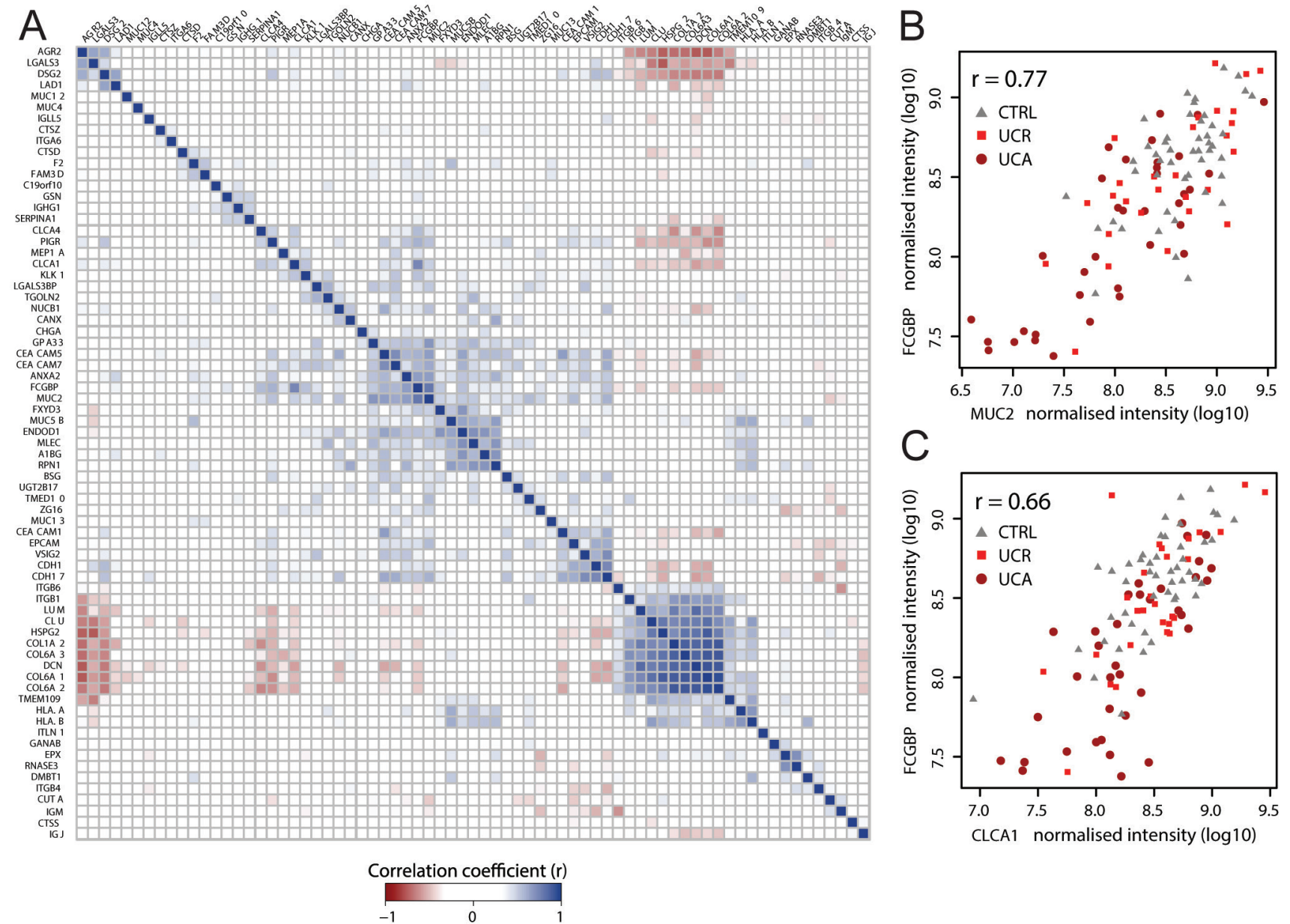

Figure 2 Correlations between the mucus proteins. (A) Matrix of Pearson $r$ correlation coefficients for all members of the mucus proteome (71 proteins), based on their normalised intensities in all patient samples $(n=111)$. Correlation of normalised intensities of (B) MUC2 and FCGBP $(r=0.77$; $\mathrm{p}<0.0001)$ and $(\mathrm{C})$ FCGBP and CLCA1 $(r=0.66 ; \mathrm{p}<0.0001)$. Patients with active UC (UCA) and UC in remission (UCR) are denoted as circles and squares, respectively; controls (CTRL) as triangles.

mucus proteins. ${ }^{22} 23$ However, when the mucus abundance of proteins from three intracellular locations (nucleus, mitochondrion and cytoplasm), as well as major blood components, was compared between patient categories, there were no discernible differences (online supplementary figure S6A,B). The concentration of mucus core proteins is higher in the inner layer than the outer, according to studies in mice. ${ }^{2}$ Here, the thickness of the impervious inner layer did not differ between patient categories (online supplementary figure S6C). ${ }^{69}$

To further validate our observations, absolute quantification of MUC2 was undertaken for 10 patients with UC (five with active disease) and five controls (online supplementary tables S3 and S8). The median MUC2 concentration was 378 molecules/fl mucus (IQR: 106-454) in active UC, as compared with 638 molecules/ fl (IQR: 437-785) in controls (online supplementary figure S6D). We concluded that the reduction in mucus structural components in active UC represents a true pathophysiological phenomenon.

\section{The reduction in mucus core proteins precedes inflammation and is associated with interleukin 18 activation}

Next, we asked whether tissue damage secondary to inflammation could explain the lower concentrations of mucus structural components in active UC. To address this question, the patients with UC were restratified based on histological scoring of biopsies from the same segment as those used for mucus collection. This resulted in three distinct categories: clinical and histological remission $(n=23)$ and clinically active with $(n=15)$ or without $(n=18)$ inflammation of the sigmoid colon (table 1, online supplementary table S9). Local inflammation was defined as Sandborn histological score $\geq 2 .{ }^{8}$ Full results of the proteomic analysis for these patient groups are provided in the online supplementary table S10. Interestingly, the reduction of MUC2 and FCGBP in active UC was equally pronounced in mucus from non-inflamed tissue (figure 4A), even from areas with Sandborn score 0; that is, a normal-appearing mucosa, including a normocellular lamina propria (online supplementary figure S7A). ${ }^{8}$ The absence of local inflammation in these patients was confirmed through normal mucus levels of calprotectin and other immune cell-derived proteins (eg, LCP1, IgM) in the proteomic analysis. These proteins were, as expected, significantly elevated in samples from inflamed tissue (figure 4B, online supplementary figure S7B). Furthermore, MUC2 and FCGBP levels did not correlate with the extent of infiltration of different immune cell populations in the lamina propria (online supplementary table S11).

However, when the mucus proteome was mined for inflammatory mediators, the abundances of peptides from the bioactive part of interleukin 18 (IL18) were found to be globally increased in active UC, with no difference between samples from inflamed versus non-inflamed tissue. By contrast, the levels of peptides 
Table 2 Members of the colon core mucus proteome and their abundance differences between patient groups

\begin{tabular}{|c|c|c|c|c|c|}
\hline \multirow[b]{2}{*}{ Protein name } & \multirow[b]{2}{*}{ Gene } & \multirow[b]{2}{*}{ Suggested role in the mucus layer } & \multicolumn{2}{|c|}{ Differentially regulated (P value) } & \multirow[b]{2}{*}{ Rankt } \\
\hline & & & UCA versus CTRL* & UCR versus CTRL* & \\
\hline Anterior gradient protein 2 & AGR2 & Required for mucin biosynthesis & n.s. (n.s.) & n.s. (n.s.) & 30 \\
\hline Chromogranin A & CHGA & Secreted by enteroendocrine cells & $\downarrow 0.032(0.006)$ & n.s. (n.s.) & 196 \\
\hline CLCA1 & CLCA1 & Major component in mucus & $\downarrow 0.011(0.004)$ & n.s. (n.s.) & 18 \\
\hline Cathepsin D & CTSD & Secreted cysteine protease & n.s. (n.s.) & n.s. (n.s.) & 530 \\
\hline Cathepsin S & CTSS & Secreted cysteine protease & n.s. (n.s.) & n.s. (n.s.) & 734 \\
\hline Cathepsin Z & CTSZ & Secreted cysteine protease & $\downarrow 0.006$ (n.s.)‡ & n.s. (n.s.) & 349 \\
\hline Decorin & $D C N$ & Proteoglycan, part of extracellular matrix & n.s. (n.s.) & n.s. (n.s.) & 211 \\
\hline DMBT1 & DMBT1 & Aggregates both $\mathrm{G}+/$ - bacteria & n.s. $($ n.s. $)$ & n.s. (n.s.) & 277 \\
\hline Eosinophil peroxidase & $E P X$ & Eosinophil granule protein & $\uparrow 0.006(0.007)$ & n.s. (n.s.) & 505 \\
\hline Protein FAM3D & FAM3D & Chemotaxis & n.s. (n.s.) & n.s. (n.s.) & 695 \\
\hline IgGFc-binding protein & FCGBP & Structural mucus protein & $\downarrow<0.001(<0.001) \ddagger$ & n.s. (n.s.) & 19 \\
\hline Cell surface $\mathrm{A} 33$ antigen & GPA33 & Affects intestinal permeability & $\downarrow 0.019(0.015)$ & n.s. (n.s.) & 296 \\
\hline $\lg A$ & IGHA1 & $\lg A$ & n.s. (n.s.) & n.s. (n.s.) & 24 \\
\hline $\operatorname{lgJ}$ & IGJ & Dimerisation of $\lg A$ & n.s. (n.s.) & n.s. (n.s.) & 61 \\
\hline $\lg M$ & IGHM & $\lg M$ & n.s. $(0.042) \uparrow$ & n.s. (n.s.) & 203 \\
\hline Intelectin-1 & ITLN1 & Found in mucus granules & n.s. (n.s.) & n.s. (n.s.) & 387 \\
\hline Kallikrein-1 & KLK1 & Serine protease found in mucus granules & 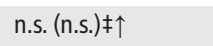 & n.s. (n.s.) & 586 \\
\hline Galectin-3 & LGALS3 & Lectin-binding galactose & n.s. (n.s.) & n.s. (n.s.) & 60 \\
\hline Galectin-3-binding protein & $\angle G A L S 3 B P$ & Galectin-3-binding protein & n.s. $(0.010) \downarrow$ & n.s. (n.s.) & 747 \\
\hline Lumican & LUM & Proteoglycan, part of extracellular matrix & n.s. (n.s.) & n.s. (n.s.) & 143 \\
\hline Meprin alpha & MEP1A & Metalloprotease & n.s. (n.s.) & n.s. (n.s.) & 917 \\
\hline Mucin-2 & MUC2 & Intestinal mucin & $\downarrow<0.001(<0.001) \ddagger$ & n.s. (n.s.) & 13 \\
\hline Mucin-4 & MUC4 & Transmembrane mucin & n.s. (n.s.) & n.s. (n.s.) & 755 \\
\hline Mucin-5B & MUC5B & Gel-forming mucin & n.s. (n.s.) & n.s. (n.s.) & 367 \\
\hline Mucin-12 & MUC12 & Transmembrane mucin & n.s. (n.s.) & n.s. (n.s.) & 363 \\
\hline Mucin-13 & MUC13 & Transmembrane mucin & n.s. (n.s.) & n.s. $(0.010) \uparrow$ & 561 \\
\hline Polymeric Ig receptor & PIGR & Transports IgA & n.s. (n.s.) & n.s. (n.s.) & 76 \\
\hline Eosinophil cationic protein & RNASE3 & Eosinophil granule protein & $\uparrow 0.010$ (n.s.) $\ddagger$ & $\uparrow 0.003(0.010)$ & 490 \\
\hline Zymogen granule protein 16 & ZG16 & Aggregates G+ bacteria & $\downarrow 0.001(0.002)$ & n.s. (n.s.) & 262 \\
\hline
\end{tabular}

*Arrows denote a decrease/increase of proteins in UCA or UCR versus CTRL, the numbers denote $p$ values. P values outside the bracket refer to the clinical stratification of patients with UC based on a synthesis of clinical Mayo score, endoscopy Mayo score and histology. Values inside the brackets refer to patients who were active/in remission based on sigmoid histology (Sandborn scale, cut-off for inflammation $\geq 2$ ), and exclude patients who were clinically active but did not have inflammation of the sigmoid colon $(n=18)$. Comparisons were performed by the Mann-Whitney $\mathrm{U}$ test.

†Abundance rank in the total identified proteome.

¥Significantly altered in patients with UC with clinical activity without local active inflammation in the sigmoid colon (Sandborn scale $\leq 1$ ), as compared with the control group.

CTRL, controls; n.S., not significant; UCA, active UC; UCR, UC in remission.

from the N-terminal fragment found in the inactive IL18 proform did not differ from controls (figure 4C). IL18 can be activated by inflammasome-mediated proteolytic cleavage, in epithelial or haematopoietic cells. ${ }^{24}$ Thus, our observations suggest that epithelial inflammasome activation and weakening of the mucus barrier precede immune cell recruitment in UC pathogenesis.

\section{Goblet cell secretory response to microbes is impaired in active UC due to a reduction in sentinel goblet cells}

Having excluded inflammation and epithelial injury as the cause of our findings, we turned to the regulation of mucus secretion. Goblet cells are a heterogeneous population. Surface cells secrete mucus constitutively, with high turnover. ${ }^{25}$ By contrast, certain upper crypt goblet cells respond to microbial challenge by a coordinated expulsion of whole mucus granules, orchestrated by a distinct cell population denoted as sentinel goblet cells. ${ }^{1326}$ Sentinel goblet cells guard the crypt openings by mounting an NLRP6 inflammasome-mediated response to TLR ligands, triggering a compound exocytosis reaction which spreads sequentially to adjacent, responsive goblet cells. ${ }^{13}$ Simultaneously, the sentinel goblet cell is expelled into the mucus. $^{13}$
Here, we used the TLR2 ligand $\mathrm{P}_{3} \mathrm{CSK}_{4}$ to stimulate sigmoid colon biopsies from controls $(n=5)$, patients with UC with active disease ( $\mathrm{n}=5$; all with local inflammation), and in remission $(\mathrm{n}=5)$. Mucus growth significantly increased in biopsies from controls and patients in remission following apical $\mathrm{P}_{3} \mathrm{CSK}_{4}$ stimulation, whereas in active UC this response was abrogated (figure 4D). Alcian blue/ periodic acid-Schiff staining of unstimulated biopsies from the same individuals showed that the crypt goblet cells of patients with active UC still contained mucus (online supplementary figure S8). By contrast, the number of sentinel goblet cells was markedly reduced in active UC (figure 4E,F). Our observations indicate that the goblet cell secretory defence system is in a refractory state in active UC, likely because of a reduction in sentinel goblet cells due to repeated microbial stimulation.

\section{Mucus penetrability was linked to proteins associated with mucin barrier formation and goblet cell responses to microbial challenge}

The inner mucus layer of patients with active UC tends to be abnormally penetrable. ${ }^{6}$ Therefore, mucus penetrability to bacteria-sized fluorescent beads was analysed in parallel for a subset of participants $(49 / 111 ; 44 \%)$. This method correlates closely 

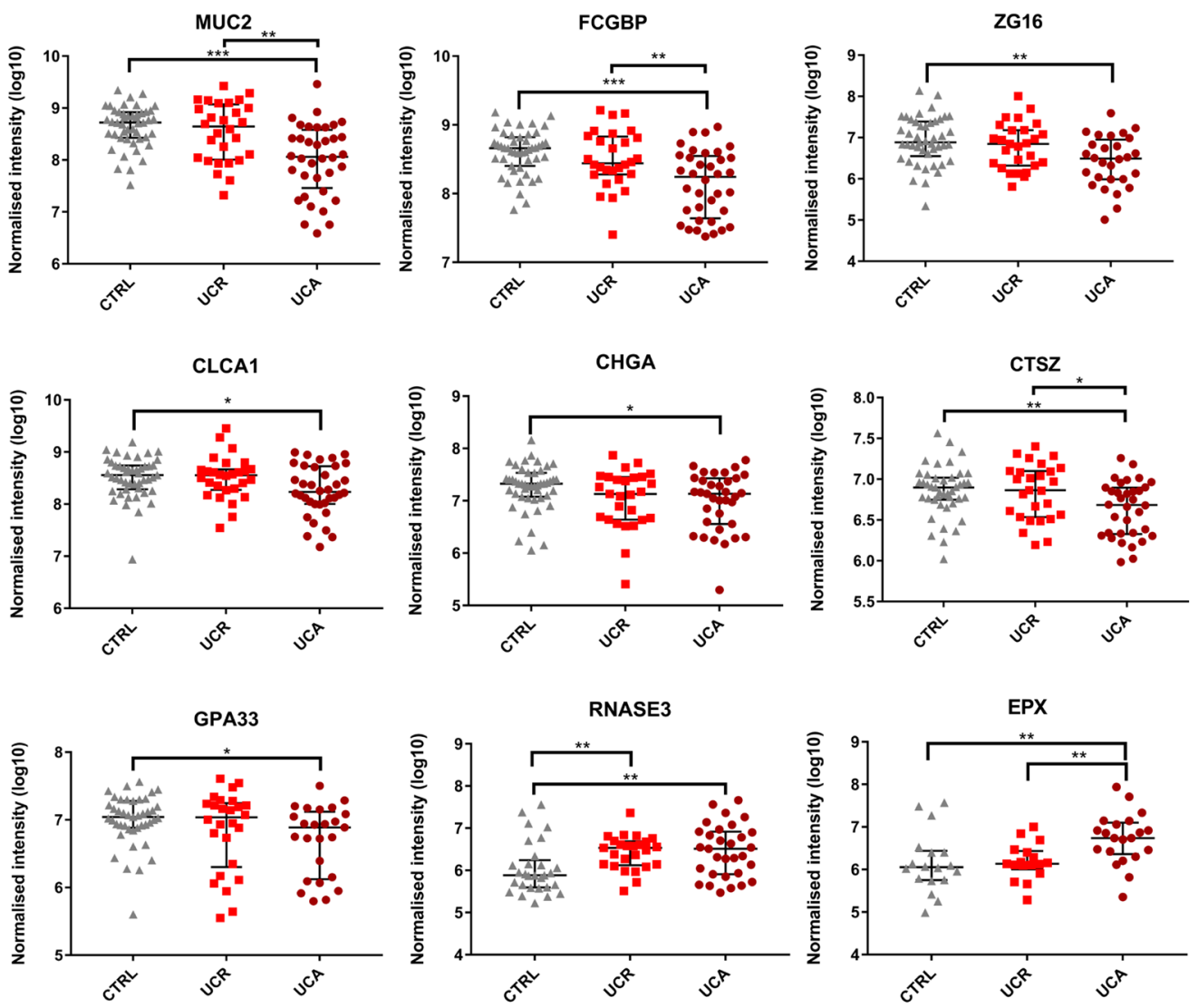

Figure 3 Core mucus proteins that were significantly altered in active UC. Data for the individual patients are overlaid by the median and IQR. Significance was determined by one-way analyses of variance (ANOVA) with Tukey's post hoc test, followed by pairwise Mann-Whitney $U$ tests $\left({ }^{*} \mathrm{p} \leq 0.05 ;{ }^{*} \mathrm{p} \leq 0.01 ;{ }^{* *} \mathrm{p} \leq 0.001\right)$. CTRL, controls; UCA, active UC; UCR, UC in remission.

with $16 \mathrm{~S}$ fluorescence in situ hybridisation staining for bacteria in the mucus, and is less affected by prior laxative treatment. ${ }^{6}$ The inner layer was (semi)penetrable in $9 / 25$ (36\%) patients with active UC versus 3/15 (20\%) patients in remission, and 1/9 (11\%) controls (figure $5 \mathrm{~A})$. The proportion of penetrable samples in this study was lower than previously reported for active UC, likely since biopsies were overwhelmingly obtained from non-inflamed or mildly affected tissue. ${ }^{6}$ Confocal microscopy images of inner mucus layer defined as impenetrable, semipenetrable and penetrable are exemplified in figure $5 \mathrm{~B}$; representative $\mathrm{z}$-stack images of all (semi)penetrable mucus samples are compiled in the online supplementary figure $S 9$.

Although MUC2 levels tended to be lower (figure 5C) in semi/ penetrable mucus, none of the mucus core proteins were significantly altered. However, lower abundance of putative mucus granule wall components, including BCAP31 and RAB10, was observed in penetrable mucus (figure 5D). ${ }^{27}$ These proteins lack a signal sequence for secretion, but would be expelled into the mucus during compound exocytosis of whole mucus granules. $^{1326}$ BCAP31 and RAB10 levels correlated closely with MUC2 in patients with UC but not controls (figure 5E), in line with the expected enhanced importance of this secretion mechanism in challenged or inflamed tissue.

Furthermore, a reduction of chloride-bicarbonate exchanger SLC26A3 (DRA, downregulated in adenoma) was observed in penetrable mucus from patients with UC (figure 5F). ${ }^{28}{ }^{29}$ Interestingly, this decrease was more pronounced in penetrable mucus from patients with UC in remission rather than during active inflammation (figure $5 \mathrm{G}$ ), hinting at a possible causative mechanism for a subgroup of patients with UC. SLC26A3 is normally expressed at the apical membrane of colonocytes and surface goblet cells (figure $5 \mathrm{H}$ ). Complementary immunostaining revealed reduced membrane expression of SLC26A3 in patients with UC with penetrable mucus (figure 5I,J), with a strong correlation between mucus levels and apical membrane staining intensity (figure $5 \mathrm{~K}$ ). As bicarbonate is critical for mucin network formation, a reduction in SLC26A3 may result in a defective mucus barrier. ${ }^{29} 30$

\section{DISCUSSION}

The intestinal mucus is our most extensive interface towards the surrounding environment. In the small intestine, the mucus network is loose and permeable, allowing for the passage of nutrients. By contrast, in colon a filter-like mucus structure limits exposure to the abundant luminal microbiota. ${ }^{2}$ Inappropriate immune activation by commensal microbiota is thought to underlie UC pathogenesis, and could result from an abnormally penetrable mucus barrier. ${ }^{16}$ However, because of the challenges of collecting and preserving the transparent and highly hydrated mucus, its composition and potential role in UC pathogenesis have evaded investigation.

In this large-scale proteomic study, we describe the colonic mucus barrier composition and how it is altered in UC. MS analysis of mucus collected ex vivo from biopsies revealed that the colonic mucus is formed by around 30 core proteins. Of these, MUC2 and FCGBP are important structural components, 


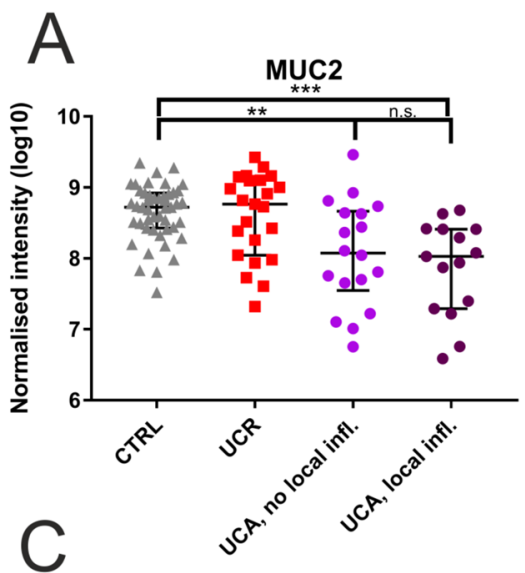

IL18 active form
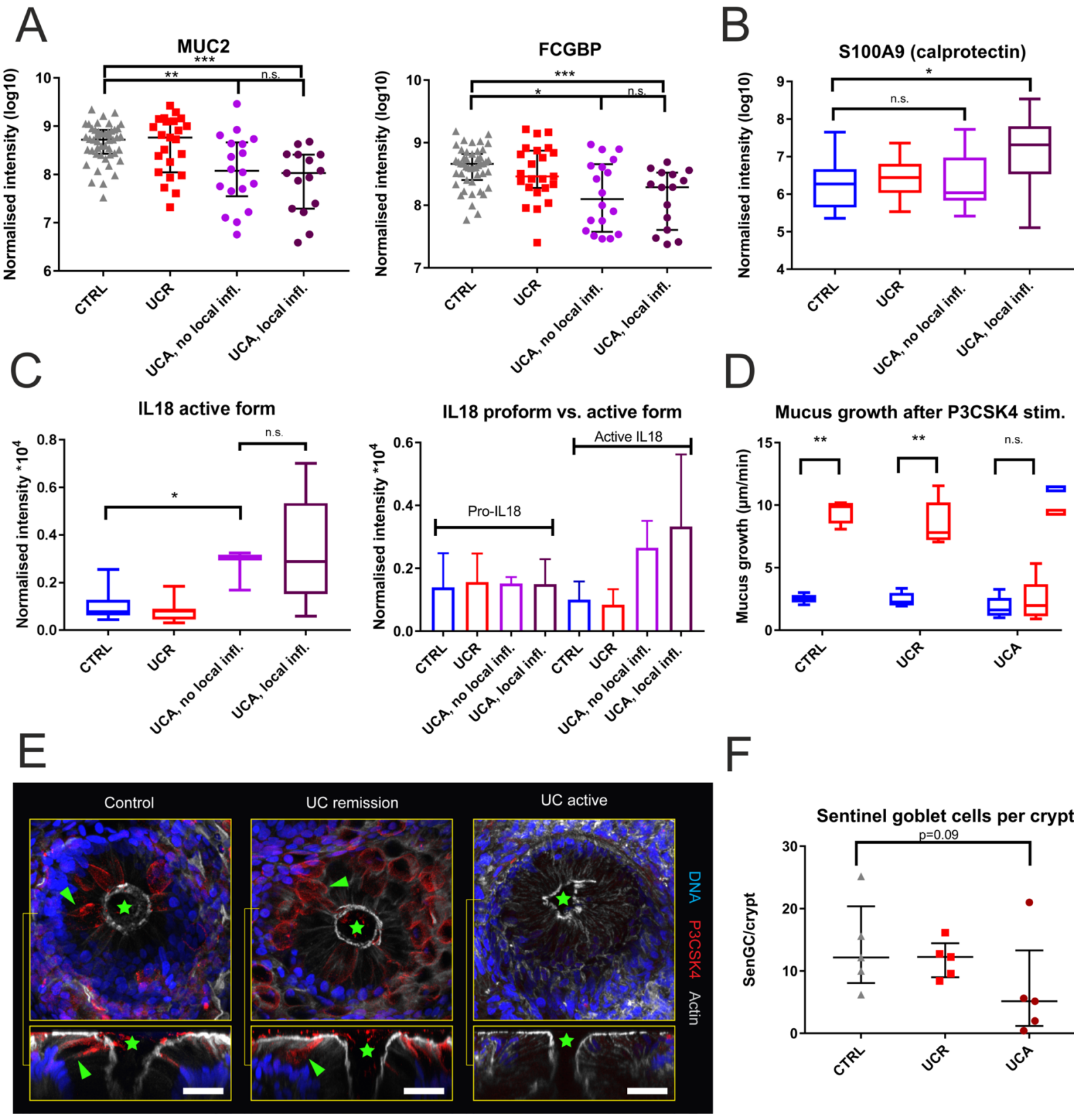

Mucus growth after P3CSK4 stim.
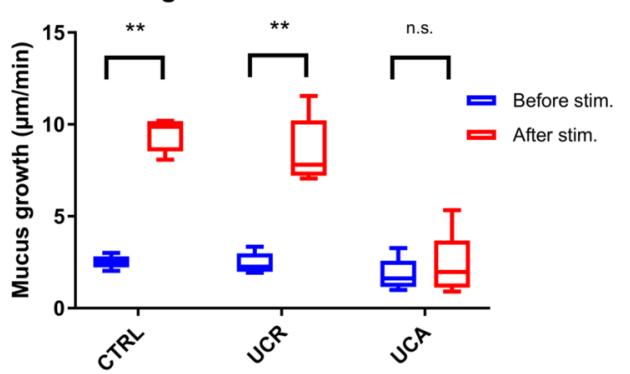

F
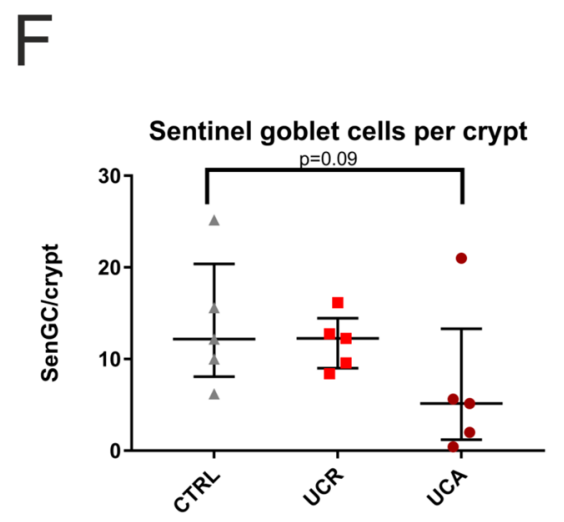

Figure 4 The reduction in mucus core proteins is not directly caused by inflammation, but likely related to exhaustion of the upper crypt goblet cell secretory response to microbes. (A) FCGBP and MUC2 levels did not differ between patients with clinically active UC with and without inflammation of the sigmoid colon. Data are overlaid by the median and IQR. Inflammation was defined as a Sandborn score $\geq 2$. (B) Mucus levels of the neutrophil protein S100A9, part of the calprotectin dimer, were not increased in patients with clinically active UC without local inflammation. (C) Mucus levels of the active part of IL18 were equally increased in patients with clinically active UC with and without local inflammation. Quantification based on two unique peptides (SDIIFFQR and SIMFTVQNED.-). The levels of the inactive IL18 propeptide did not differ between patient groups, based on one unique peptide (AAEPVEDNCINFVAMK). Bars represent the mean; error bars 1 SD. One control that was diagnosed with colon cancer during follow-up was excluded from analysis. (D) Sentinel goblet cell secretory response to the bacterial Toll-like receptor (TLR) 1/2-ligand $\mathrm{P}_{3} \mathrm{CSK}_{4}$ was abrogated in active UC. The box plot shows data from five controls, five patients with UC in remission and five patients with active UC. (E) The number of sentinel goblet cells (red) was reduced in patients with active UC. The upper panel shows cross sections (x/y axis view) of a representative sigmoid colon biopsy from each patient category; the lower panel longitudinal (x/z axis) views. Green stars represent crypt openings; green arrowheads represent examples of sentinel goblet cells. Sentinel goblet cells were identified as upper crypt goblet cells that had endocytosed fluorescently labelled $P_{3} C_{\text {CSK }}$. Scale bars: $30 \mu \mathrm{m}$. (F) Mean number of sentinel goblet cells per crypt for each patient category. Individual observations are overlaid with median and IQR. The high outlier among the patients with active UC had commenced peroral cortisone treatment 3 weeks prior to colonoscopy. For all box plots, the line represents the median, the box the IQR and the whiskers the range. Groups were compared by the Mann-Whitney U test with Bonferroni correction for multiple comparisons. CTRL, controls; IL18, interleukin 18; n.S., not significant; UCA, active UC; UCR, UC in remission.

whereas ZG16 and DMBT1 aggregate and trap bacteria. 2162021 However, contrary to previous assumptions, classical antimicrobial proteins were not detected. ${ }^{18} 19$ Presumably, the healthy colon, given its impervious inner mucus layer, does not require these proteins for mucosal defence. Instead, they could be deleterious for the mutualistic host-microbial relationship. 
A

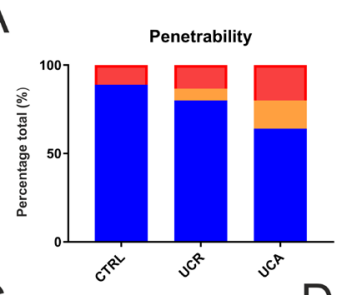

C

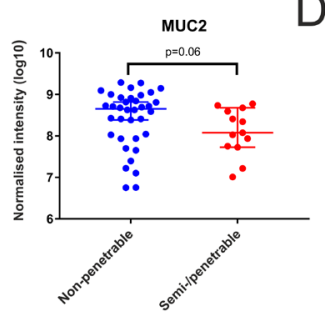

E
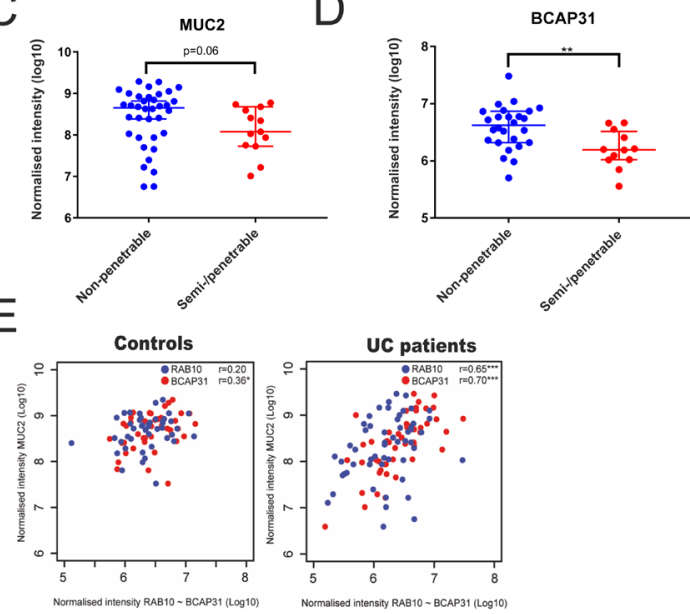

F
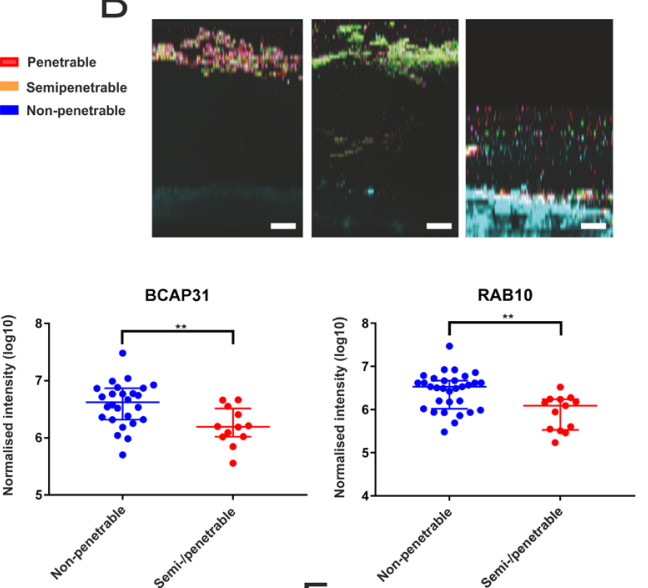

SLC26A3 in all UC patients

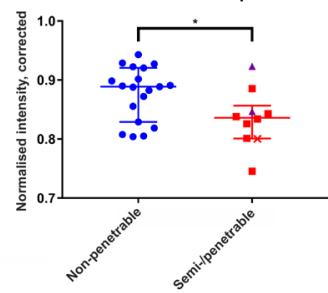

G

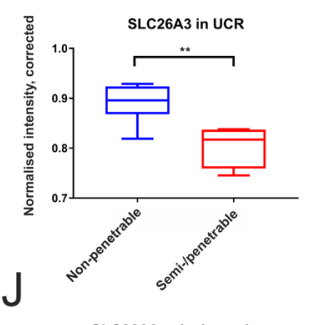

$\mathrm{H}$
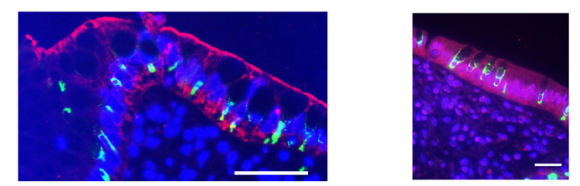

$\mathrm{K}$

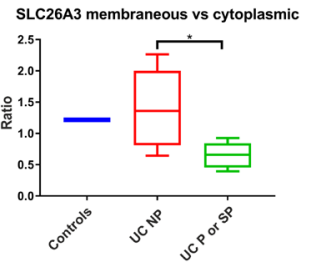

Figure 5 Changes in mucus protein composition associated with inner mucus layer failure. (A) Proportions of penetrable, semipenetrable and non-penetrable mucus samples among patients with UC and controls. (B) Representative confocal images of (from left to right) impenetrable, semipenetrable and penetrable mucus. The blue colour represents the epithelium; red, purple and green dots represent fluorescent beads with diameters of $0.5,1$ and $2 \mu \mathrm{m}$, respectively. (C) MUC2 levels in non-penetrable and semi/penetrable mucus. (D) Levels of putative compound exocytosis markers BCAP31 and RAB10 in non-penetrable and semi/penetrable mucus. (E) Correlations of normalised intensities between MUC2 levels and BCAP31 and RAB10, respectively, for patients with UC and for the control group. $R$ values refer to the Spearman's rank correlation coefficient. (F) Levels of chloride-bicarbonate transporter SLC26A3 (DRA) were reduced in semi/penetrable mucus from patients with UC. Triangles represent two patients with active disease who regained an impenetrable inner mucus layer in remission, whereas the $\mathrm{x}$ represents a patient in remission, whose mucus remained penetrable upon reanalysis after 3 years. (G) The reduction in mucus SLC26A3 was particularly pronounced in individuals in remission. One borderline case with clinical Mayo score 2, endoscopic Mayo score 1 and Sandborn score 1 was included among the remission patients. In (F) and (G), SLC26A3 abundance factors were corrected for the degree of mucus cellular contamination through the division of SLC26A3 normalised intensity values by the median normalised intensity of the top 100 most abundant intracellular proteins, for each sample. (H) SLC26A3 (red) was apically localised in both colonocytes and surface goblet cells. Costaining of non-O-glycosylated apomucin 2 (localised to the endoplasmic reticulum [ER]; green) identifies goblet cells; nuclei are stained blue. Scale bar: $50 \mu \mathrm{m}$. (I) In patients with UC with penetrable mucus, membrane staining for SLC26A3 (red) appeared weaker. Scale bar: $25 \mu \mathrm{m}$. (J) The left panel shows a comparison of the intensity of SLC26A3 apical membrane staining for patients with UC with and without penetrable mucus. The right panel shows the ratio between membrane and cytoplasmic SLC26A3 staining intensity for each patient category. Results are based on immunohistochemical analysis of patients with UC with (semi)penetrable mucus $(n=6)$, patients with UC with non-penetrable mucus $(n=6)$ and controls $(n=3)$, and represent averages of 15 measurements. (K) Correlation between SLC26A3 apical membrane staining intensity and SLC26A3 mucus levels according to relative quantification by proteomics. The $r$ value refers to the Spearman's rank correlation coefficient. For all box plots, the line represents the median, the box the IQR and the whiskers the range of values. Groups were compared by the Mann-Whitney U test. ${ }^{*} \mathrm{p} \leq 0.05 ;{ }^{*} \mathrm{p} \leq 0.01$; ${ }^{* *} \mathrm{p} \leq 0.001$. CTRL, controls; NP, non-penetrable; SP, semipenetrable; $\mathrm{P}$, penetrable; UCA, active UC; UCR, UC in remission. 
The most striking finding of this study was a reduction of mucus core structural components in patients with UC with ongoing inflammation. The inner mucus layer tends to be abnormally penetrable to bacteria in active UC. ${ }^{6}$ Here we report that its main structural components, MUC2 and FCGBP, are less abundant in patients with active inflammation. Moreover, other goblet cell products that may be important for innate immunity, including ZG16, were also reduced. ${ }^{21}$

In an attempt to delineate the temporal and causal relationship between our observations and UC inflammation, we studied mucus samples from a large number of patients with clinical activity, but without involvement of the sampled segment. Intriguingly, the reduction in mucus structural components was equally pronounced in these samples, as in mucus from inflamed tissue. Thus, structural weakening of the mucus layer occurs independent of, and likely prior to, inflammation.

To determine the initiating mechanisms behind the observed mucus abnormalities and the onset of disease activity, we screened the mucus proteomes for mediators of early inflammation. In contrast to classical immune cell-derived markers, the levels of the bioactive part of IL18 were globally increased in mucus from patients with active UC, also from non-inflamed segments. This proinflammatory cytokine is activated by caspase 1 -mediated cleavage following inflammasome assembly in epithelial or haematopoietic cells. ${ }^{24}$ Given the apparent lack of immune cell recruitment in many biopsies, epithelial inflammasome activation would be the most likely source of the secreted IL18.

Colonic mucus secretion is a highly sophisticated process. Intercrypt goblet cells constitutively secrete mucus, through the fusion of single granules with the membrane. ${ }^{25}$ Goblet cells in the upper portion of the crypts, by contrast, accumulate mucus that is secreted in a massive, coordinated burst on sensing of microbes. ${ }^{132631}$ This process involves the release of whole mucus granules, and is known as compound exocytosis. ${ }^{31}$ Compound exocytosis of mucus is orchestrated by a distinct cell population denoted as sentinel goblet cells. ${ }^{13}$ These cells sense bacterial components and products through several TLRs. Endocytosis of the TLR ligand triggers NLRP6 inflammasome-dependent mucus release. This reaction is transmitted to adjacent, responsive goblet cells through juxtacrine gap junction signalling. Ultimately, the sentinel goblet cell is expelled into the mucus. Thus, these cells act as protective guardians of the crypt openings. ${ }^{13}$

To assess whether sentinel goblet cell-dependent secretion is deficient in UC, biopsies were stimulated ex vivo with TLR1/2 ligand $\mathrm{P}_{3} \mathrm{CSK}_{4}$. In biopsies from controls and patients in remission, mucus growth rapidly increased after $\mathrm{P}_{3} \mathrm{CSK}_{4}$ administration. However, in active UC this response was abrogated. Moreover, there was a marked reduction in sentinel goblet cell numbers in active UC. Taken together, our observations indicate that epithelial exposure to TLR ligands triggers inflammasome assembly during the initial phase of a UC flare, resulting in activation of the compound exocytosis response. However, with repeated or continuous microbial challenge, attenuation of this mechanism occurs, due to insufficient time for replenishment of the sentinel goblet cell population. These findings are consistent with results from short-term dextran sodium sulphate (DSS) treatment of mice, where epithelial inflammasome activation, sentinel goblet cell depletion and mucus barrier failure precede overt inflammation. ${ }^{13}$

Protein markers for the different modes of secretion could be helpful in studying their importance for barrier function. BCAP31 and RAB10 are known mucus granule membrane components that lack a signal peptide for secretion. ${ }^{27}$ Therefore, they would only be expelled during compound exocytosis of whole granules, making them putative markers for microbiota-induced mucus secretion. BCAP31 and RAB10 levels correlated closely with MUC2 in patients with UC, but not in controls. This observation underlines the importance of compound exocytosis as a secondline defence in challenged or inflamed mucosa. Strikingly, both proteins were significantly reduced in penetrable mucus.

Collectively, our observations suggest a model for the initiating events during a UC flare, where increased host-bacterial contact and secondary epithelial inflammasome activation lead to hyperstimulation of mucus secretion. This may result in sentinel goblet cell depletion and ensuing attenuation of microbiota-induced mucus secretion, triggering a vicious circle of increased epithelial exposure to bacteria, aggravated inflammation and further breakdown of the mucus barrier.

While compositional mucus abnormalities probably occur prior to overt inflammation, the nature of the 'first hit' remains unelucidated. Since one-fifth of patients with UC in remission had penetrable mucus, primary mucus barrier defects might conceivably be the underlying factor for a subset of patients. One clue to a causative mechanism could be the reduction of the apical membrane chloride-bicarbonate transporter SLC26A3 observed in patients with UC with penetrable mucus, particularly in the absence of active inflammation. Several loss-of-function mutations in SLC26A3 have been described, with the common clinical manifestation of chloride-rich diarrhoea. ${ }^{28}$ However, since bicarbonate is required for luminal MUC2 network formation, reduced expression of SLC26A3 could also result in a defective mucus barrier. ${ }^{29} 30$ Accordingly, $\mathrm{SLC}_{26 \mathrm{~A}} 3^{-/}$mice lack a stratified inner mucus layer, and are highly susceptible to induced colitis. ${ }^{29}$ The SLC26A3 gene has previously been identified as a UC risk locus. ${ }^{32}$ Our observations indicate that this association may be explained by a defective inner mucus layer.

Interestingly, mucus structural components were not altered in UC in remission. However, there was a significant upsurge in eosinophil proteins. Tissue eosinophil infiltration in quiescent disease has been suggested to promote mucosal healing. ${ }^{33}$ Still, toxic eosinophil products, such as RNASE3, might conceivably contribute to symptoms during remission periods, including concomitant IBS which is more common in patients with UC than the general population. ${ }^{34}$

Some limitations of our study merit consideration. Due to physiological differences, mucus protein composition might vary slightly between the anatomical segments of the large intestine. ${ }^{35}$ However, as UC typically starts distally, studies of sigmoid colon should be highly relevant. ${ }^{1}$ Furthermore, bowel preparation and the ex vivo regrowth and collection method could potentially affect mucus composition. Nevertheless, these methodological caveats are likely to primarily influence cellular contamination, for which there was no evidence of bias between patient groups. Third, the control group consisted of patients referred for colonoscopy due to various symptoms or findings. Still, none of these individuals had any observable macroscopic or microscopic colon pathology, except for diverticulosis and minor polyps which can be regarded as normal population variants.

In conclusion, our observations indicate that compositional alterations of the colonic mucus are common in active UC. While the temporal and causal relationships remain incompletely understood, our findings suggest that mucus barrier weakening is associated with exhaustion of the sentinel goblet cell response to microbial challenge. The observed mucus abnormalities were not secondary to local inflammation, suggesting that they may instead precede and contribute to activation of the disease. Our investigation provides a platform for further studies 
of the underlying mechanisms and their exploitation as putative therapeutic targets.

Correction notice This article has been corrected since it published Online First. The legend for figure 1 has been amended.

Acknowledgements The authors thank Lisbeth Eklund and Karin Ahlman for help with biopsy and mucus collection. The authors also thank all the helpful colonoscopists at the Endoscopy Unit of the Sahlgrenska University Hospital for providing biopsies.

Contributors Design of studies: SvdP, KSJ, GB, MEVJ, GCH. Performance of experiments: SvdP, KSJ, GB, LA, NA. Clinical assessments: KSJ, HS. Writing of manuscript: SvdP, KSJ, GCH. All authors approved the manuscript.

Funding This work was supported by the National Institute of Allergy and Infectious Diseases (U01AI095473), Knut and Alice Wallenberg Foundation, European Research Council (ERC), Swedish Research Council, Swedish Cancer Foundation, IngaBritt and Arne Lundberg Foundation, Sahlgrenska University Hospital (ALF), Wilhelm and Martina Lundgren Foundation and Adlerbert Research Foundation.

Disclaimer The content is solely the responsibility of the authors and does not necessarily represent the official views of the $\mathrm{NIH}$.

Competing interests None declared.

Patient consent for publication Not required.

Ethics approval The study protocol was approved by the regional ethics committee.

Provenance and peer review Not commissioned; externally peer reviewed.

Open access This is an open access article distributed in accordance with the Creative Commons Attribution Non Commercial (CC BY-NC 4.0) license, which permits others to distribute, remix, adapt, build upon this work non-commercially, and license their derivative works on different terms, provided the original work is properly cited, appropriate credit is given, any changes made indicated, and the use is non-commercial. See: http://creativecommons.org/licenses/by-nc/4.0/.

ORCID iD

Gunnar C Hansson http://orcid.org/0000-0002-1900-1869

\section{REFERENCES}

1 Danese S, Fiocchi C. Ulcerative colitis. N Engl J Med 2011;365:1713-25.

2 Johansson ME, Phillipson M, Petersson J, et al. The inner of the two Muc2 mucindependent mucus layers in colon is devoid of bacteria. Proc Natl Acad Sci U S A 2008:105:15064-9.

3 Van der Sluis M, De Koning BA, De Bruijn AC, et al. Muc2-deficient mice spontaneously develop colitis, indicating that MUC2 is critical for colonic protection. Gastroenterology 2006;131:117-29.

4 Velcich A, Yang W, Heyer J, et al. Colorectal cancer in mice genetically deficient in the mucin Muc2. Science 2002;295:1726-9.

5 Blumberg RS, Saubermann LJ, Strober W. Animal models of mucosal inflammation and their relation to human inflammatory bowel disease. Curr Opin Immunol 1999:11:648-56.

6 Johansson ME, Gustafsson JK, Holmén-Larsson J, et al. Bacteria penetrate the normally impenetrable inner colon mucus layer in both murine colitis models and patients with ulcerative colitis. Gut 2014;63:281-91.

7 Schroeder KW, Tremaine WJ, Ilstrup DM. Coated oral 5-aminosalicylic acid therapy for mildly to moderately active ulcerative colitis. A randomized study. N Engl J Med 1987;317:1625-9.

8 Sandborn WJ, Tremaine WJ, Schroeder KW, et al. A placebo-controlled trial of cyclosporine enemas for mildly to moderately active left-sided ulcerative colitis. Gastroenterology 1994:106:1429-35.

9 Gustafsson JK, Ermund A, Johansson ME, et al. An ex vivo method for studying mucus formation, properties, and thickness in human colonic biopsies and mouse small and large intestinal explants. Am J Physiol Gastrointest Liver Physiol 2012;302:G43 0-G438.
10 Gallien S, Duriez E, Crone C, et al. Targeted proteomic quantification on quadrupoleorbitrap mass spectrometer. Mol Cell Proteomics 2012:11:1709-23.

11 Cox J, Neuhauser N, Michalski A, et al. Andromeda: a peptide search engine integrated into the MaxQuant environment. J Proteome Res 2011:10:1794-805.

12 MacLean B, Tomazela DM, Shulman N, et al. Skyline: an open source document editor for creating and analyzing targeted proteomics experiments. Bioinformatics 2010;26:966-8.

13 Birchenough GM, Nyström EE, Johansson ME, et al. A sentinel goblet cel guards the colonic crypt by triggering Nlrp6-dependent Muc2 secretion. Science 2016:352:1535-42.

14 Bergström JH, Berg KA, Rodríguez-Piñeiro AM, et al. AGR2, an endoplasmic reticulum protein, is secreted into the gastrointestinal mucus. PLoS One 2014:9:e104186.

15 Anderson NL, Polanski M, Pieper R, et al. The human plasma proteome: a nonredundant list developed by combination of four separate sources. Mol Cell Proteomics 2004;3:311-26.

16 Johansson ME, Thomsson KA, Hansson GC. Proteomic analyses of the two mucus layers of the colon barrier reveal that their main component, the Muc2 mucin, is strongly bound to the Fcgbp protein. J Proteome Res 2009;8:3549-57.

17 Podolsky DK, Lynch-Devaney K, Stow JL, et al. Identification of human intestinal trefoil factor. Goblet cell-specific expression of a peptide targeted for apical secretion. J Biol Chem 1993;268:6694-702.

18 Meyer-Hoffert U, Hornef MW, Henriques-Normark B, et al. Secreted enteric antimicrobial activity localises to the mucus surface layer. Gut 2008;57:764-71.

19 Bevins CL, Salzman NH. Paneth cells, antimicrobial peptides and maintenance of intestinal homeostasis. Nat Rev Microbiol 2011:9:356-68.

20 Rodríguez-Piñeiro AM, Bergström JH, Ermund A, et al. Studies of mucus in mouse stomach, small intestine, and colon. II. Gastrointestinal mucus proteome reveals Muc2 and Muc5ac accompanied by a set of core proteins. Am J Physiol Gastrointest Liver Physiol 2013;305:G348-G356.

21 Bergström JH, Birchenough GM, Katona G, et al. Gram-positive bacteria are held at a distance in the colon mucus by the lectin-like protein ZG16. Proc Natl Acad Sci U SA 2016;113:13833-8.

22 Iwamoto M, Koji T, Makiyama K, et al. Apoptosis of crypt epithelial cells in ulcerative colitis. J Pathol 1996:180:152-9.

23 Heazlewood CK, Cook MC, Eri R, et al. Aberrant mucin assembly in mice causes endoplasmic reticulum stress and spontaneous inflammation resembling ulcerative colitis. PLoS Med 2008;5:e54.

24 Nowarski R, Jackson R, Gagliani N, et al. Epithelial IL-18 Equilibrium Controls Barrier Function in Colitis. Cell 2015;163:1444-56.

25 Johansson ME. Fast renewal of the distal colonic mucus layers by the surface goblet cells as measured by in vivo labeling of mucin glycoproteins. PLoS One 2012:7:e41009.

26 Grootjans J, Hundscheid IH, Lenaerts K, et al. Ischaemia-induced mucus barrier loss and bacterial penetration are rapidly counteracted by increased goblet cell secretory activity in human and rat colon. Gut 2013;62:250-8.

27 Rodríguez-Piñeiro AM, van der Post S, Johansson ME, et al. Proteomic study of the mucin granulae in an intestinal goblet cell model. J Proteome Res 2012;11:1879-90.

28 Höglund P, Haila S, Socha J, et al. Mutations of the Down-regulated in adenoma (DRA) gene cause congenital chloride diarrhoea. Nat Genet 1996;14:316-9.

29 Xiao F, Yu Q, Li J, et al. SIc26a3 deficiency is associated with loss of colonic HCO3 (-) secretion, absence of a firm mucus layer and barrier impairment in mice. Acta Physiol 2014;211:161-75

30 Ambort D, Johansson ME, Gustafsson JK, et al. Calcium and pH-dependent packing and release of the gel-forming MUC2 mucin. Proc Natl Acad Sci U S A 2012:109:5645-50.

31 Specian RD, Neutra MR. Mechanism of rapid mucus secretion in goblet cells stimulated by acetylcholine. J Cell Biol 1980:85:626-40.

32 Khor B, Gardet A, Xavier RJ. Genetics and pathogenesis of inflammatory bowel disease. Nature 2011:474:307-17.

33 Lampinen M, Rönnblom A, Amin K, et al. Eosinophil granulocytes are activated during the remission phase of ulcerative colitis. Gut 2005;54:1714-20.

34 Isgar B, Harman M, Kaye MD, et al. Symptoms of irritable bowel syndrome in ulcerative colitis in remission. Gut 1983:24:190-2.

35 van der Post S, Hansson GC. Membrane protein profiling of human colon reveals distinct regional differences. Mol Cell Proteomics 2014;13:2277-87. 